\title{
Basic Guidelines in Writing a Scientific Article
}

\author{
Alexandru Florin Rogobete \\ Clinic of Anesthesia and Intensive Care, "Pius Brinzeu" Emergency County Hospital, Timișoara, Romania
}

Medicine is a complex multidisciplinary domain, which combines a lot of branches of science such as biology, genetics, biochemistry, physiology etc. Medical science has evolved a lot lately in the field of clinical or therapeutic pharmacology, in patient monitoring and in regard to interventional surgery, mainly due to the intense research carried out in these domains. Worldwide, in medical research centers, daily impressive studies are taking place. One of the most important aspects in regard to dissemination of the scientific information gained by researches, is represented by the publication of these studies in the form of scientific articles in renowned international journals. ${ }^{1}$

For a scientific article to be successful, and above it to be useful to the readers worldwide, it should respect a series of guidelines, more or less known to the general public. Scientific articles are divided into many categories, such as a scientific report type of article, original article, review article, short communication or editorial. ${ }^{2}$ One of the most important types of articles, most widely searched by the publishers of the journals as well as by the readers, are the original articles. The structure of the article comprises of Abstract, Introduction, Materials and methods, Results, Discussion and References. In this editorial, we would like to present briefly several "secrets" that can prove beneficial in designing a scientific article. ${ }^{3}$

The abstract represents practically the summary of the article. For original articles, the abstract is structured in the form of Introduction, Aims, Material and methods, Results, and Conclusions. The abstract should be written with great attention, as many times this is the only part of the article which is read by editors or by the readers. For mak- ing the article-writing work easy, it is recommended to begin with writing the Material and methods section first. In this section of the article, detailed information should be provided regarding the "recipe" of the study. It is very important, that in this section we clearly introduce the place where the study took place, time frame when the study was conducted, who comprised the study lot, as well as who was not included in the study group. Similarly, it is important to mention the approval of the institute's ethic commission, permitting the study to take place. Practically, the Material and methods section should answer the following questions: when, where, how and on whom was the study conducted. Similarly, in this section sufficient information for the study to be productive for other researchers, should be mentioned. ${ }^{4}$ On one hand we should present what we know and how we performed it, and on the other hand we should mention what we obtained. And this is how the Results section arises. In this section, one should write the results obtained following the protocol described in the Methods section. It is very important, to discuss exactly and precisely what was done.,5 Results not related to the study should not be included in this section. It is recommended to use tables and figures, in order to present the information more clearly. Once this section is finalized, we get a complete picture of the study. We know what we did, where and how we did it, and what we got as the end-product. Now comes the moment to write the introduction and the discussions. In the Introduction information stating the importance of the topic of the study should be touched, and a short description of the subject should be given a baseline. Further, in this section objectives of the study should be mentioned along 
with their importance in connection to the results. It is recommended that the Introduction should not exceed 500 words. The Discussions section should practically be in the form of arguments and should discuss strictly the results obtained in the study. It is not recommended to write the literature in details. Many times, the discussions are confused with a review of the literature, which is not permitted for an original article. Every part of the results obtained should be argued and compared to that obtained by similar studies. In this section, it is recommended to quote other authors, other studies, to support and solidify the message and content of the article. Furthermore, it is recommended to mention the limitations of the study. Many times, authors think that mentioning the limitations of the study will make the article weak to be published by the editors of the journals, and so they avoid it. But this is a complete mistake, as transparently presenting the facts concerning the limitations of the research work, increases the worth and credibility of the article, permitting the readers or researchers to understand more clearly the results of the study. ${ }^{5,6}$

The Conclusions must highlight the findings and their importance. It is not recommended to include in this section other information, that is not related to the study.

An important section is represented by the references. These practically sustain the study, making a series of connections between the various studies having the same common subject. References should be written respecting a uniform format, as per the guidelines specified by each journal. $^{7}$

In conclusion we can confirm that writing a scientific article is moreover an art. Designing such an article should combine scientific transparency, imagination and the ability to summarize objective scientific information and its scientific significance along with the message it transmits.

\section{REFERENCES}

1. Kramer B, Libhaber E. Writing for publication: institutional support provides an enabling environment. BMC Med Educ. 2016;16(1):115.

2. Dumache R, Ciocan V, Muresan C, Enache A. Molecular DNA Analysis in Forensic Identification. Clin Lab. 2016;1-4.

3. Florek AG, Dellavalle RP. Case reports in medical education: a platform for training medical students, residents, and fellows in scientific writing and critical thinking. J Med Case Rep. 2016;10(1):86.

4. Marta MM. Brief History of the Evolution of the Medical Research Article. Clujul Med. 2015;88(4):567.

5. Hulede IV, Chang A. Inside ASM Education ASM Scientific Writing and Publishing Institute: Program Impacts and Future Expansion. J Microbiol Biol Educ. 2015;16(2):136-137.

6. Danka ES, Malpede BM. Reading, Writing, and Presenting Original Scientific Research: A Nine-Week Course in Scientific Communication for High School Students. J Microbio/ Biol Educ. 2015;16(2):203-210.

7. Zhang $\mathrm{C}$, Zhu Y, The B, Table F, He H, Central B. China' s graduate students need better education in scientific writing and publishing. J Zhejiang Univ-Sci B. 2016;17(5):409-412. 